Research Article

\title{
Factorizable Ordered Hypergroupoids with Applications
}

\author{
Xiaolong Shi, ${ }^{1}$ Hao Guan $\mathbb{D D}^{1}{ }^{1}$ Maryam Akhoundi, ${ }^{2}$ and Saber Omidi $\mathbb{D}^{3}$ \\ ${ }^{1}$ Institute of Computing Science and Technology, Guangzhou University, Guangzhou 510006, China \\ ${ }^{2}$ Clinical Research Development Unit of Rouhani Hospital, Babol University of Medical Sciences, Babol, Iran \\ ${ }^{3}$ Ministry of Education Iran, Department of Education, Tehran, Iran
}

Correspondence should be addressed to Hao Guan; guanhao@gzhu.edu.cn

Received 31 July 2021; Revised 18 September 2021; Accepted 4 October 2021; Published 18 October 2021

Academic Editor: Yong Aaron Tan

Copyright (C) 2021 Xiaolong Shi et al. This is an open access article distributed under the Creative Commons Attribution License, which permits unrestricted use, distribution, and reproduction in any medium, provided the original work is properly cited.

In this study, we propose the concept of factorizable ordered hypergroupoids (semihypergroups) and present several of its properties. Our goal is to construct ordered hypergroupoid from blood groups together with some other information. Finally, we discuss right magnifying elements for further research.

\section{Introduction and Preliminaries}

Marty [1] proposed the notion of a hypergroup based on the multivalued operations. Hyperstructures have many applications in both pure and applied sciences. Some researchers have tried to discuss important biological phenomena in the framework of fuzzy hyperstructures. Hyperstructures were used in many disciplines such as theoretical physics, coding theory, and biology. Basic concepts and relevant applications concerning hyperstructure theory can be found in $[2,3]$.

Ordered semihypergroups are suggested by Heidari and Davvaz [4] and then investigated by Davvaz et al. in [5] (also, see [6-8]). The research about generalization of hyperideals in ordered hyperstructures is growing rapidly $[9,10]$. In recent years, pseudoorders have received extensive attention in ordered hyperstructures $[5,8]$. Using the notion of (weak) pseudoorder [5, 8], several examples of ordered semi (hyper) groups have been constructed in connection with ordered semihypergroups. Weak pseudoorders can remarkably support the constructions of ordered semihypergroups [8].

Breakable semihypergroups were firstly presented by Heidari and Cristea [11] in 2019. In a breakable semihypergroup, each nonempty subset is a subsemihypergroup. The cyclicity of the EL-hyperstructures is expressed in [12]. Recently, much attention has been paid to investigating the factorizable hyperstructures. In [13], Heidari and Cristea have suggested the concept of factorizable semihypergroups by using the concept of factorizable semigroups [14]. In this regard, Munir et al. [15] initiated the study of factorizable hypergroupoids and discussed their properties. For future work, one could extend the existing works $[13,15]$ to the framework of fuzzy sets.

In this note, we offer basic concepts on factorizable ordered hypergroupoids. We show that if a right hyperideal $R$ and a left hyperideal $L$ form the factors of an ordered hypergroupoid $S$, then $R \cap L=(R \diamond L]$. Connection between regular and factorizable ordered semihypergroups is presented. Moreover, we prove that if an ordered hypergroupoid contains either a right (left) magnifying element, then it is factorizable.

Now, we describe several information on an ordered hypergroupoid (semihypergroup) $(S, \diamond, \leq)$.

A hyperoperation is a mapping $\diamond: S \times S \longrightarrow P^{*}(S)$, where $P^{*}(S)$ denotes the family of all nonempty subsets of $S$. The couple $(S, \diamond)$ is called a hypergroupoid. If $\varnothing \neq U, V \subseteq S$ and $x \in S$, then

$$
\begin{aligned}
& U \diamond V=\underset{\substack{u \in U \\
v \in V}}{\bigcup} u \diamond v, \\
& x \diamond U=\{x\} \diamond U, \\
& U \diamond x=U \diamond\{x\} .
\end{aligned}
$$


A semihypergroup is a hypergroupoid $(S, \diamond)$, where

$$
(u \diamond v) \diamond w=u \diamond(v \diamond w), \quad \text { for all } u, v, w \in S .
$$

A semihypergroup $S$ is a hypergroup if the reproduction axiom is verified as

$$
x \diamond S=S=S \diamond x, \quad \text { for all } x \in S .
$$

A hyperoperation $\diamond$ on $\varnothing \neq S$ is extensive [12] if $\{a, b\} \subseteq a \diamond b$ for all $a, b \in S$.

Definition 1 (see $[4,5]$ ). A hypergroupoid $(S, \diamond)$ is called an ordered hypergroupoid (semihypergroup) if

(1) $(S, \leq)$ is an ordered set

(2) $u \leq v$ implies $u \diamond w \prec v \diamond w$ and $w \diamond u \prec w \diamond v$, for all $u, v, w \in S$

If $\varnothing \neq A, B \subseteq S$, then

$$
A \prec B \Leftrightarrow \forall a \in A, \quad \exists b \in B ; a \leq b .
$$

Here, $\varnothing \neq K$ of an ordered hypergroupoid (semihypergroup) $(S, \diamond, \leq)$ is called a subhypergroupoid (subsemihypergroup) of $S$ if $a \diamond b \subseteq K$ for every $a, b \in K$ and $\leq_{K}$ is the relation $\leq$ restricted to $K$. Set

$$
(I]:=\{x \in S \mid x \leq i \quad \text { for some } i \in I\} .
$$

If $\varnothing \neq I, J \subseteq S$, then
(1) $I \subseteq(I]$
(2) If $I \subseteq J$, then $(I] \subseteq(J]$
(3) $((I] \diamond(J]]=(I \diamond J]$

An ordered hypergroupoid $(S, \diamond, \leq)$ is regular if $w \in(w \diamond S \diamond w]$ for every $w \in S$.

Definition 2 (see $[4,5])$. A set $\varnothing \neq K$ of an ordered hypergroupoid $(S, \diamond, \leq)$ is left (resp. right) hyperideal if

(1) $S \diamond K \subseteq K($ resp. $K \diamond S \subseteq K)$

(2) $(K] \subseteq K$

Here, a hyperideal is a left hyperideal of $S$ being right hyperideal.

\section{Results and Discussion}

We extend the definition in [15] to the ordered case. In this section, we pay attention to the factorizable ordered hypergroupoids that is obligatory to prove our proposing results.

Definition 3. An ordered hypergroupoid (semihypergroup) $(S, \diamond, \leq)$ is said to be factorizable if there exist two different proper subhypergroupoids (subsemihypergroups) $U$ and $V$ of $S$ such that $S=(U \diamond V]$. The pair $(U, V)$ is called a factorization of $S$, with factors $U$ and $V$.
Example 1. Consider an ordered hypergroup $S=\{R, W\}$ with the following hyperoperation $\diamond$ (as shown in Table 1) and (partial) order relation $\leq$.

$$
\leq:=\{(R, R),(W, W)\} .
$$

The subhypergroups $\{R\}$ and $\{W\}$ then form the factorization of $S$ as

$$
(\{R\} \diamond\{W\}]=(S]=S .
$$

There are four main categories within the $A B O$ blood group system: $A, B, O$, and $A B$. The detail of blood groups is presented in $[15,16]$, and here we will not repeat them again. An application of ordered hypergroupoid to blood groups is given.

Example 2. The ABO blood groups are giving in a set

$$
S=\{A, B, A B, O\} .
$$

Define the hyperoperation $\diamond$ (as shown in Table 2 ) and (partial) order relation $\leq$ on $S$ as follows.

$$
\begin{aligned}
\leq & :=\{(O, O),(O, A),(O, B),(O, A B),(A, A),(A, A B), \\
& (B, B),(B, A B),(A B, A B) .
\end{aligned}
$$

Then, $(S, \diamond, \leq)$ is an ordered hypergroupoid. The covering relation of $S$ is given by

$$
\prec=\{(O, A),(O, B),(A, A B),(B, A B)\} .
$$

The Hasse diagram of $S$ is shown in Figure 1.

It is easily seen that

(1) Clearly, $O \leq A \leq A B$ and $O \leq B \leq A B$. From the figure, it can be obviously seen that $O$ is the donor blood group and $A B$ is the recipient blood group. Also, $A \leq A B$ shows that people with blood group $A$ can donate blood to people with blood groups $A$ and $A B$. Similarly, $B \leq A B$ shows that people with blood group $B$ can donate blood to people with blood groups $B$ and $A B$.

(2) The subhypergroupoids $U=\{A, O\}$ and $V=\{B, O\}$ form the factorization of $S$ as $(U \diamond V]=(S]=S$.

(3) $S$ is a simple ordered hypergroupoid. It can be seen that $S$ is simple if and only if $S=(S \diamond x \diamond S$ ] for every $x \in S$.

Theorem 1. Let $(S, \diamond, \leq)$ be an ordered hypergroupoid. If the right hyperideal $R$ and the left hyperideal $L$ form the factors of $S$, then

$$
R \cap L=(R \diamond L]
$$

Proof. As $R \diamond L \subseteq S \diamond L \subseteq L$ and $R \diamond L \subseteq R \diamond S \subseteq R$, we have $(R \diamond L] \subseteq(R \cap L]$. Now, we obtain 
TABle 1: Codominant alleles of a gene.

\begin{tabular}{lcc}
\hline$\diamond$ & $R$ & $W$ \\
\hline$R$ & $R$ & $\{R, W\}$ \\
$W$ & $\{R, W\}$ & $W$ \\
\hline
\end{tabular}

TABLE 2: $A B O$ blood group inheritance.

\begin{tabular}{lcccc}
\hline$\diamond$ & $A$ & $B$ & $A B$ & $O$ \\
\hline$A$ & $\{A, O\}$ & $S$ & $\{A, B, A B\}$ & $\{A, O\}$ \\
$B$ & $S$ & $\{B, O\}$ & $\{A, B, A B\}$ & $\{B, O\}$ \\
$A B$ & $\{A, B, A B\}$ & $\{A, B, A B\}$ & $\{A, B, A B\}$ & $\{A, B\}$ \\
$O$ & $\{A, O\}$ & $\{B, O\}$ & $\{A, O\}$ & $O$ \\
\hline
\end{tabular}

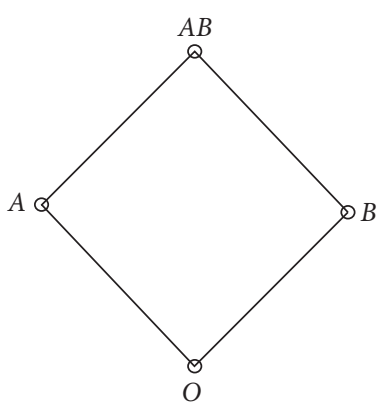

FIgURE 1: Figure of $S$ for Example 1.

$$
\begin{aligned}
(R \diamond L] & \subseteq(R \cap L] \\
& \subseteq(R] \cap(L] \\
& =R \cap L .
\end{aligned}
$$

We need to show that $R \cap L \subseteq(R \diamond L]$. Since $(R, L)$ is a factorization of $S$, it follows that $(R \diamond L]=S$. It implies that $R \cap L \subseteq S=(R \diamond L]$.

Theorem 2. If $(S, \diamond, \leq)$ is an ordered hypergroup, then $S$ is regular.

Proof. Take any $x \in S$. Then,

$$
(x \diamond S]=S=(S \diamond x] .
$$

So, $x \leq x \diamond a$ for some $a \in S$. Again, $a \leq b \diamond x$ for some $b \in S$. Thus,

$$
x \leq x \diamond a \leq x \diamond(b \diamond x) .
$$

It implies that $x \in(x \diamond S \diamond x]$. Hence, $S$ is regular.

Theorem 3. Let $(S, \diamond, \leq)$ be an ordered semihypergroup, where $\leq:=\{(x, y) \mid x=y\}$. Let $S$ be factorizable as $S=(U \diamond V]$, where $U$ is a group and $|u \diamond v|=1$ for all $u \in U$ and $v \in V$. If $V$ is a regular ordered semihypergroup, then $S$ is also a regular ordered semihypergroup.

Proof. Take any $x \in S=(U \diamond V]$. Then, $x \leq u \diamond v$ for some $u \in U$ and $v \in V$. By assumption, $V$ is regular. Consider the element $v \in V$ and assume that $v \leq v \diamond v^{\prime} \diamond v$ for some $v^{\prime} \in V$. So,

$$
\begin{aligned}
x & \leq u \diamond v \\
& \leq u \diamond\left(v \diamond v^{\prime} \diamond v\right) \\
& \leq u \diamond v \diamond v^{\prime} \diamond\left(u^{-1} \diamond u\right) \diamond v \\
& =(u \diamond v) \diamond v^{\prime} \diamond u^{-1} \diamond(u \diamond v) .
\end{aligned}
$$

Thus, $x \leq x \diamond w \diamond x$ for some $w \in v^{\prime} \diamond u^{-1} \subseteq S$. Hence, $S$ is regular.

Corollary 1. Let $(S, \diamond, \leq)$ be an ordered semihypergroup, where $\leq:=\{(x, y) \mid x=y\}$. Let $S$ be factorizable as $S=(U \diamond V]$, where $U$ is a group and $|u \diamond v|=1$ for all $u \in U$ and $v \in V$. If $V$ is an ordered hypergroup, then $S$ is regular.

Proof. By Theorem 2, $V$ is a regular ordered semihypergroup. Now, by Theorem $3, S$ is also regular.

An ordered hypergroupoid $(S, \diamond, \leq)$ is said to be extensive if $\{a, b\} \subseteq a \diamond b$ for all $a, b \in S$.

Corollary 2. Let $(S, \diamond, \leq)$ be an ordered semihypergroup, where $\leq:=\{(x, y) \mid x=y\}$. Let $S$ be factorizable as $S=(U \diamond V]$, where $U$ is a group and $|u \diamond v|=1$ for all $u \in U$ and $v \in V$. If $V$ is an extensive ordered semihypergroup, then $S$ is regular.

Proof. Let $V$ be an extensive ordered semihypergroup. Then, $\{a, b\} \subseteq a \diamond b$ for all $a, b \in S$. So,

$$
t \diamond S=\bigcup_{s \in S} t \diamond s=S
$$

for all $t \in S$. Hence, $S$ is an ordered hypergroup. Now, by Corollary $1, S$ is regular.

Definition 4. An element $x$ of an ordered hypergroupoid $(S, \diamond, \leq)$ is said to be a right (resp. left) magnifying element if there exists a proper subhypergroupoid $U$ of $S$ such that $S=(U \diamond x]($ resp. $S=(x \diamond U])$.

Example 3. In Example 2, $U=\{A, O\}$ is a subhypergroupoid of $S$. We have

$$
\begin{aligned}
(U \diamond\{A B\}] & =(\{A, O\} \diamond\{A B\}]=(\{A, B, A B\}]=S, \\
(U \diamond\{B\}] & =(\{A, O\} \diamond\{B\}]=(S]=S .
\end{aligned}
$$

Thus, $A B$ and $B$ are right magnifying elements of $S$. We consider next the subhypergroupoid $V=\{B, O\}$. Observe that if $V=\{B, O\}$, then $A B$ and $A$ are right magnifying elements of $S$.

Example 4. Given a hypergroup $S=\{a, b, c, d\}$, let the hyperoperation $\diamond$ be defined in Table 3 .

Consider an ordered hypergroup $(S, \diamond, \leq)$ with the following (partial) order relation $\leq$ : 
TABLE 3: Table of $\diamond$ for Example 4.

\begin{tabular}{ccccc}
\hline$\diamond$ & $a$ & $b$ & $c$ & $d$ \\
\hline$a$ & $a$ & $a$ & $\{a, b, c\}$ & $\{a, b, d\}$ \\
$b$ & $a$ & $a$ & $\{a, b, c\}$ & $\{a, b, d\}$ \\
$c$ & $\{a, b, c\}$ & $\{a, b, c\}$ & $\{a, b, c\}$ & $\{c, d\}$ \\
$d$ & $\{a, b, d\}$ & $\{a, b, d\}$ & $\{c, d\}$ & $\{a, b, d\}$ \\
\hline
\end{tabular}

$$
\begin{aligned}
\leq & :=\{(a, a),(a, b),(a, c),(a, d),(b, b),(b, c),(b, d), \\
& (c, c),(c, d),(d, d) .
\end{aligned}
$$

Note that based on the data from Table $3, U=\{a, b, c\}$ is a subhypergroup of $S$. If $x=d$, then

$$
(U \diamond x]=(\{a, b, c\} \diamond d]=(S]=S .
$$

Hence, $d$ is a right (left) magnifying element of $S$.

An ordered hypergroupoid $(S, \diamond, \leq)$ is called cyclic, if there exists a generator $x \in S$ such that for all $s \in S$, there exists $n \in \mathbb{N}$ such that $s \in x^{n}$.

Proposition 1. Let $(S, \diamond, \leq)$ be an ordered hypergroupoid. If $S$ contains either a right (left) magnifying element $x$, then $S$ is factorizable.

Proof. We shall prove this for right magnifying element $x$. We consider the following cases.

Case 1. Let $S$ be noncyclic.

If $x$ is a right magnifying element of $S$, then $S=(U \diamond x]$, where $U$ is a proper subhypergroupoid of $S$. Since $S$ is noncyclic, it follows that $S$ is factorizable because $S=(U \diamond V]$, where $V=\langle x\rangle$.

Case 2. Let $S$ be cyclic and $S=\langle a\rangle$.

As $x \in S$ is a right magnifying element, $S=(U \diamond x]$, where $U$ is a proper subhypergroupoid of $S$. Since $U$ is a proper subhypergroupoid of $S$, we get $U=\left\langle a^{k}\right\rangle$, where $k \in \mathbb{N}$. Moreover, for $l \in \mathbb{N}$, let $x=a^{l}$. Hence,

$$
\begin{aligned}
S & =(U \diamond x] \\
& =\left(\left\langle a^{k}\right\rangle \diamond a^{l}\right] \\
& =\left(\left\langle a^{k+l}\right\rangle\right] \\
& =\left(\left\langle a^{m}\right\rangle\right],
\end{aligned}
$$

where $m=k+l$, which is a contradiction because $\left(\left\langle a^{m}\right\rangle\right]$ is a proper subhypergroupoid of $S$. Now, the proof is completed.

Definition 5. An ordered hypergroupoid (semihypergroup) $(S, \diamond, \leq)$ is said to be generalized factorizable if there exist two different proper subsets $U$ and $V$ of $S$ such that $S=(U \diamond V]$. The pair $(U, V)$ is called a generalized factorization of $S$, with generalized factors $U$ and $V$.
Example 5

(1) Consider the ordered hypergroupoid $S=\{A, B, A B, O\}$ defined in Example 2. The subsets $\{A\}$ and $\{B\}$ then form the generalized factorization of $S$ as

$$
(\{A\} \diamond\{B\}]=(S]=S .
$$

Thus, $S$ can be composed by the parents possessing only $A$ and $B$ blood groups.

(2) Consider the ordered hypergroup $S=\{a, b, c, d\}$ defined in Example 4. The subsets $\{a, c\}$ and $\{b, d\}$ then form the generalized factorization of $S$ as

$$
(\{a, c\} \diamond\{b, d\}]=(S]=S .
$$

\section{Conclusions}

In this paper, we have described factorizable ordered hypergroupoids. We have also shown some results in this respect. An application into the fields of blood groups and factorizable ordered hypergroupoid theory was briefly introduced. We finished our study with generalized factorizable ordered hypergroupoids in hope that other factorizations such as $(m, n)$-factorizable ordered hypergroupoids can be discussed in the future. In the future, one can study applications of factorization in DNA coding theory.

\section{Data Availability}

No data were used to support this study.

\section{Conflicts of Interest}

The authors declare that they have no conflicts of interest.

\section{Acknowledgments}

This study was supported by the National Natural Science Foundation of China (61772376 and 62072129) and by the National Key R\&D Program of China (2019YFA0706402).

\section{References}

[1] F. Marty, "Sur une generalization de la notion de groupe," in Proceedings of the 8th Congress on Mathematical Education, Stockholm, Sweden, May 1934.

[2] M. Al Tahan and B. Davvaz, "A new relationship between intuitionistic fuzzy sets and genetics," Journal of Classification, vol. 36, no. 3, pp. 494-512, 2019.

[3] P. Corsini and V. Leoreanu, "Applications of hyperstructure theory," Advances in Mathematics, Kluwer Academic Publishers, Dordrecht, Netherlands, 2003.

[4] D. Heidari and B. Davvaz, "On ordered hyperstructures," Applied Mathematics Physics and Astronomy, vol. 73, no. 2, pp. 85-96, 2011. 
[5] B. Davvaz, P. Corsini, and T. Changphas, "Relationship between ordered semihypergroups and ordered semigroups by using pseudoorder," European Journal of Combinatorics, vol. 44, pp. 208-217, 2015.

[6] B. Davvaz, Semihypergroup Theory, Elsevier, Amsterdam, Netherlands, 2016.

[7] Z. Gu and X. Tang, "Ordered regular equivalence relations on ordered semihypergroups," Journal of Algebra, vol. 450, pp. 384-397, 2016.

[8] J. Tang, X. Feng, B. Davvaz, and X.-Y. Xie, “A further study on ordered regular equivalence relations in ordered semihypergroups," Open Mathematics, vol. 16, no. 1, pp. 168-184, 2018.

[9] S. Omidi and B. Davvaz, "Contribution to study special kinds of hyperideals in ordered semihyperrings," Journal of Taibah University for Science, vol. 11, no. 6, pp. 1083-1094, 2017.

[10] Y. Rao, P. Xu, Z. Shao, and S. Kosari, "Left $k$-bi-quasi hyperideals in ordered semihyperrings," Applied Mathematics Physics and Astronomy, vol. 83, no. 1, pp. 125-134, 2021.

[11] D. Heidari and I. Cristea, "Breakable semihypergroups," Symmetry Plus, vol. 11, no. 1, p. 100, 2019.

[12] M. Novák, Š. Křehlík, and I. Cristea, "Cyclicity in ELhypergroups," Symmetry Plus, vol. 10, no. 11, p. 611, 2018.

[13] D. Heidari and I. Cristea, "On factorizable semihypergroups," Mathematics, vol. 8, no. 7, p. 1064, 2020.

[14] F. Catino, "Factorizable semigroups," Semigroup Forum, vol. 36, no. 1, pp. 167-174, 1987.

[15] M. Munir, N. Kausar, S. Salahuddin, R. Xu, and W. Ahmad, "Hypergroupoids as tools for studying blood group genetics," International Journal of Fuzzy Logic and Intelligent Systems, vol. 21, no. 2, pp. 135-144, 2021.

[16] D. D. Farhud and M. Zarif Yeganeh, "A brief history of human blood groups," Iranian Journal of Public Health, vol. 42, no. 1, pp. 1-6, 2013. 